\title{
Variability in the systems of care supporting critical neonatal intensive care unit transitions
}

\author{
Heather C. Kaplan ${ }^{1,2} \cdot$ Erika M. Edwards $^{3,4,5} \cdot$ Roger F. Soll $^{3,5} \cdot$ Kate A. Morrow ${ }^{3}$. Jeffrey Meyers ${ }^{6} \cdot$ Wendy Timpson $^{7,8}$. \\ Howard Cohen $^{9} \cdot$ Marybeth Fry $^{10} \cdot$ Elizabeth Schierholz ${ }^{11,12} \cdot$ Madge E. Buus-Frank $^{13,14} \cdot$ Jeffrey D. Horbar ${ }^{3,5}$
}

Received: 13 December 2019 / Revised: 2 June 2020 / Accepted: 7 July 2020 / Published online: 14 July 2020

(C) The Author(s), under exclusive licence to Springer Nature America, Inc. 2020

\begin{abstract}
Objective Assess practices supporting care transitions for infants and families in the neonatal intensive care unit (NICU) using a model of four key drivers: communication, teamwork, family integration, and standardization.

Study design Single-day audit among NICUs in the Vermont Oxford Network Critical Transitions collaborative addressing policies and practices supporting the four key drivers during admission, discharge, shift-to-shift handoffs, within hospital transfers, and select changes in clinical status.

Results Among 95 NICUs, the median hospital rate of audited policies in place addressing the four key drivers were $47 \%$ (inter-quartile range (IQR) 35-65\%) for communication, 67\% (IQR 33-83\%) for teamwork, 50\% (IQR 33-61\%) for family integration, and 70\% (IQR 56-85\%) for standardization. Of the 2462 infants included, 1066 (43\%) experienced $\geq 1$ specified transition during the week prior to the audit.

Conclusions We identified opportunities for improving NICU transitions in areas of communication, teamwork, family integration, and standardization.
\end{abstract}

\section{Introduction}

Care delivery for infants and their families in the neonatal intensive care unit (NICU) is complex and involves

Supplementary information The online version of this article (https:// doi.org/10.1038/s41372-020-0720-3) contains supplementary material, which is available to authorized users.

Heather C. Kaplan

heather.kaplan@cchmc.org

1 Perinatal Institute, James M. Anderson Center for Health Systems Excellence, Cincinnati Children's Hospital Medical Center, Cincinnati, OH, USA

2 Department of Pediatrics, University of Cincinnati College of Medicine, Cincinnati, OH, USA

3 Vermont Oxford Network, Burlington, VT, USA

4 Department of Mathematics and Statistics, University of Vermont, Burlington, VT, USA

5 Department of Pediatrics, The Robert Larner, M.D. College of Medicine, The University of Vermont, Burlington, VT, USA

6 Division of Neonatology, Golisano Children's Hospital, University of Rochester Medical Center, Rochester, NY, USA numerous transitions starting with the initial transition to extrauterine life, followed by multiple cycles of acute and more convalescent care, then followed ultimately by hospital discharge. Furthermore, sick infants are often transported between and within hospitals during the protracted NICU stay. Daily transitions occur amongst caregivers, making frequent handoffs necessary. Gray et al. estimated that during a 6-month NICU stay, a baby experiences more than 300 nursing shift handoffs, in addition to the large

7 Department of Neonatology, Beth Israel Deaconess Medical Center, Boston, MA, USA

8 Department of Pediatrics, Harvard Medical School, Boston, MA, USA

9 Randall Children's Hospital, Portland, OR, USA

10 Akron Children's Hospital, Akron, OH, USA

11 Children's Hospital of Colorado, Aurora, CO, USA

12 Data Science to Patient Value, University of Colorado, School of Medicine, Anschutz Medical Campus, Aurora, CO, USA

13 Division of Neonatology, The Children's Hospital, Lebanon, NH, USA

14 The Dartmouth Institute for Health Policy and Clinical Practice at Geisel School of Medicine, Hanover, NH, USA 
number of handoffs between other types of providers [1]. Moreover, there is increasing recognition of the role of the NICU team to provide "follow through," assuring the infant and family safely transitions to a supportive community with adequate social and financial resources at discharge [24]. Each of these transitions in care is vulnerable periods, and if not well planned and orchestrated, may result in harm.

After reviewing the literature on transitions and handoffs in preparation for launching an internet-based quality improvement (QI) collaborative focused on critical transitions, we developed a key driver diagram (logic model) that proposes four main contributors to ideal critical transitions (Fig. 1). We define a critical transition as any change in an infant's site of care, significant change in the infant's status or plan of care, and/or change in the infant's care team. An ideal transition is one that is safe, timely, efficient, effective, equitable, patient- and family-centered, and socially responsible [5-7]. The key driver diagram suggests that, irrespective of the type of transition, ideal transitions are driven by highly reliable:

- Communication: Timely, direct, and standardized communication ensures that critical details are conveyed. Good communication occurs in a setting that supports a culture of respect, openness, and trust and is based upon shared goals, active listening, and bidirectional information exchange.
- Teamwork: Strong teamwork includes a shared mental model of care, shared goals that are prioritized by multidisciplinary collaboration, and shared accountability.

- Family integration: Families should be actively included as full partners in care, assuring that the plan of care is responsive to each patient and family's needs, preferences, and values.

- Standardization: Care during transitions should be standardized so that all patients receive care that maintains continuity, is evidence-based, safe, and cost effective, and ensures that each patient receives the right content of care, in the right place, at the right time, and from the right team.

There are many evidence-based, potentially better practices that support improved standardization, communication, teamwork, and family integration during critical transitions. For example, evidence-based team training interventions such as TeamSTEPPS ${ }^{\mathrm{TM}}$ or crew resource management training can be used to improve teamwork during transitions [8-12]. Similarly, communication during transitions can be significantly improved through standardization, training, and simulation [13-15] and telemedicine may be a particularly useful approach in improving communication, particularly at the time of birth [16]. In addition, change ideas from the literature on standardization and high reliability theory such as use of checklists, clinical practice guidelines, and pathways can be used to reduce
Fig. 1 Key driver diagram. Visual display of the iNICQ theory showing how communication, teamwork, family integration, and standardization drive optimal transitions.

Key Driver Diagram: Improving Critical Transitions

Primary Drivers

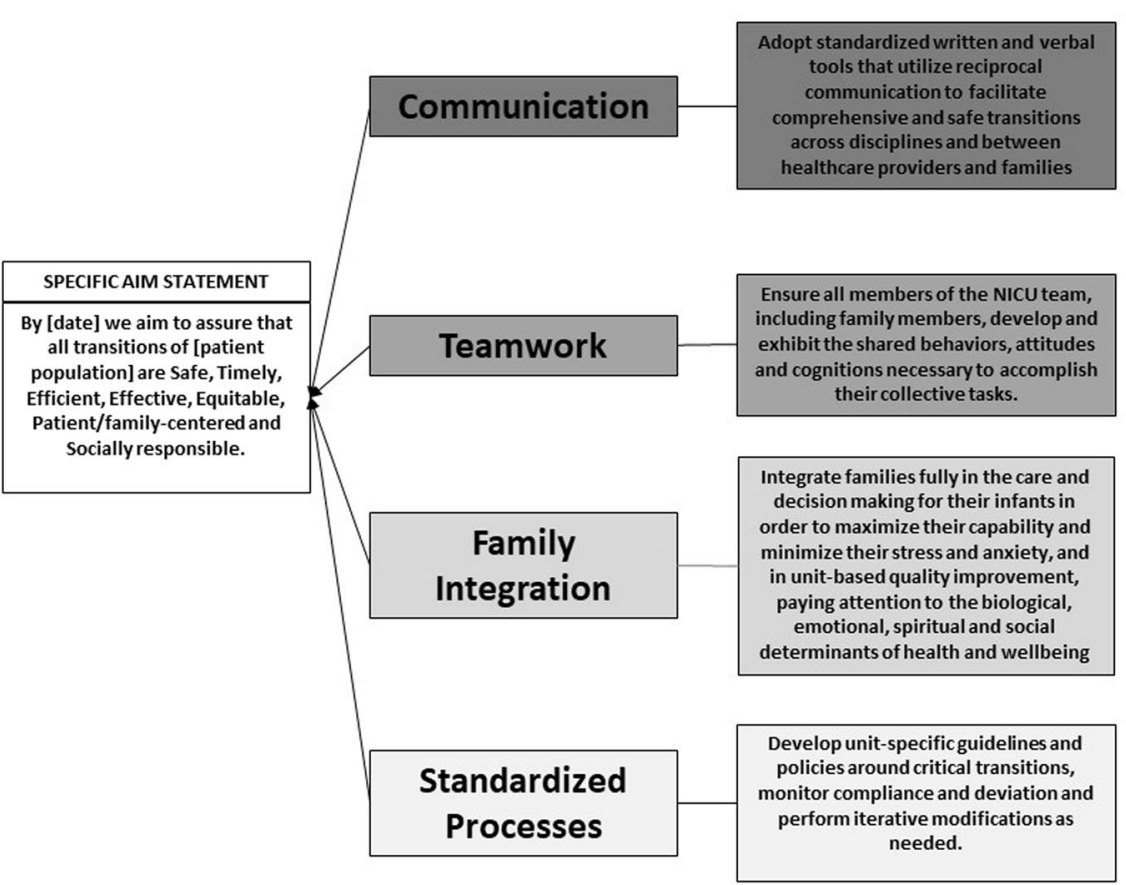


variation in care seen during clinical transitions [17-20]. Evidence from literature examining handoffs demonstrates that tools such as handoff bundles [21] and computer-based sign-out procedures can be used to reduce the risk of preventable adverse events (AEs) and errors that occur during these types of transitions [22].

In this article, our goal is to examine variability in potentially better practices related to standardization, communication, teamwork, and family integration as outlined in the project key driver diagram being used by NICUs participating in the Vermont Oxford Network (VON) Internetbased Newborn Improvement Collaborative for Quality (iNICQ), and identify the baseline prevalence of transitions in the NICU.

\section{Methods}

VON is a non-profit, voluntary worldwide collaboration dedicated to improving the quality, safety, and value of neonatal intensive care $[2,5]$. The iNICQ collaborative, Improving Critical Transitions for Every Newborn, launched in January 2019 with 122 centers participating at the time of the initial audit (Supplementary Table 1). The collaborative is focused on improving critical transitions and learning and applying experience-based co-design methodologies $[23,24]$. The global aim is to assure that all transitions are safe, timely, efficient, effective, equitable, patient/family centered, and socially responsible [5-7].

To understand practices related to transitions and to measure improvement over the course of the collaborative, a series of point-prevalence quality audits were planned, the first of which occurred in February 2019 (baseline audit). Analogous to prior VON collaboratives focused on neonatal abstinence syndrome (NAS) [25], alarm safety [26], and antibiotic stewardship [27], this audit included both unit-level and patient-level measures and occurred on a single day, selected by the local site, during a 2-week period. Auditors received a manual of operations with standardized definitions (Supplementary 2). Data on the timing of the audits (e.g., weekday vs. weekend) were not solicited.

For unit-level measures, centers were asked to respond to questions about the existence of policies, guidelines, and procedures within their unit. Decisions regarding which policies, guidelines, and procedures to include in the audit were made collectively by the authors based on their clinical expertise and the knowledge gained in reviewing the literature and formulating the key driver diagram (logic model). Patient-level audits assessed the occurrence of various types of transitions including admission (either via transport from another facility or inborn), within hospital transfers between locations or units for diagnostic tests, procedures, or surgery outside the NICU, and select changes in clinical status including initiation of antibiotics, extubation, or initiation of pharmacologic therapy for NAS within 7 days of the audit. Patient-level audits also included assessments of nursing shift-to-shift handoff and discharge preparedness, but these data are not reported in this manuscript. For patient-level audits, NICUs were given the option of auditing all infants, examining a sub-population (e.g. very low birth weight infants), or auditing a fixed sample at each interval. The audit was pilot tested by faculty prior to widespread use in the collaborative.

Questions within the audit were associated to one of the four key drivers of communication, teamwork, family integration, standardization by the authors based on their assessment of which driver the policy, process, or guideline best reflects. Data for completion of the audit came from chart review and/or interviews with bedside caregivers. Deidentified data were entered electronically through Qualtrics Research Core ${ }^{\mathrm{TM}}$ software (Provo, Utah) and were transmitted to VON.

From the 122 centers participating in iNICQ, we excluded 18 centers that reported that they did not complete the audit due to pending Institutional Review Board (IRB) determination at their local institution, 3 international centers, and 6 centers that did not report NICU infants, resulting in an aggregate sample of 95 centers (78\%) that are reported here. Descriptive statistics were used to summarize the data. To calculate the extent to which hospitals had policies and guidelines supporting each of the four key drivers, we calculated the hospital-level rate of policies and guidelines in place across the items assigned to each driver and report the minimum, 25th percentile, 50th percentile (median), 75th percentile, and maximum rates across hospitals. Characteristics to describe the participating NICUs were derived from the VON Annual Membership Survey. As this was a descriptive study without statistical comparisons, a priori power calculations were not performed.

The University of Vermont IRB determined that this project was exempt from review. Each participating center was instructed to determine whether local review was necessary and to follow their own local procedures for approval, if indicated.

\section{Results}

The characteristics of the 95 centers included in the analysis are shown in Table 1. Most centers were teaching hospitals (66\%), defined as those with neonatal fellows and/or residents participating in direct patient care in the NICU. Most centers $(67 \%)$ provided advanced levels of care, with no restriction on ventilation and the ability to perform neonatal surgery. 
Table 1 Participating unit characteristics.

\begin{tabular}{|c|c|}
\hline Characteristic & $n=95(\%)$ \\
\hline Neonatal intensive care beds, median (Q1, Q3) & $25(16,44)$ \\
\hline $\begin{array}{l}\text { Neonatal intermediate or stepdown care beds, median } \\
\text { (Q1, Q3) }\end{array}$ & $12(0,19)$ \\
\hline \multicolumn{2}{|l|}{ NICU type, $n(\%)$} \\
\hline Restrictions on ventilation $^{\mathrm{a}}$ & $2(2 \%)$ \\
\hline $\begin{array}{l}\text { No ventilation restrictions, does not perform } \\
\text { neonatal surgery }\end{array}$ & $28(30 \%)$ \\
\hline $\begin{array}{l}\text { No ventilation restrictions, performs neonatal } \\
\text { surgery except cardiac bypass }\end{array}$ & $36(38 \%)$ \\
\hline $\begin{array}{l}\text { No ventilation restrictions, performs neonatal } \\
\text { surgery including cardiac bypass }\end{array}$ & $28(30 \%)$ \\
\hline Teaching hospital, $n(\%)$ & $61(66 \%)$ \\
\hline \multicolumn{2}{|l|}{ Hospital ownership, $n(\%)$} \\
\hline Nonprofit & $66(73 \%)$ \\
\hline For profit & $11(12 \%)$ \\
\hline Government or other & $14(15 \%)$ \\
\hline \multicolumn{2}{|l|}{ Services offered, $n(\%)$} \\
\hline Therapeutic hypothermia for encephalopathy & $78(85 \%)$ \\
\hline ECMO & $32(36 \%)$ \\
\hline MRI & $91(98 \%)$ \\
\hline Inhaled nitric oxide & $86(93 \%)$ \\
\hline $24 / 7$ in-house neonatologist coverage & $53(57 \%)$ \\
\hline Family advisory council & $44(49 \%)$ \\
\hline Neurodevelopmental follow-up clinic & $85(93 \%)$ \\
\hline
\end{tabular}

${ }^{a}$ Center is required by state regulation or local hospital policy to transfer infants to another hospital for assisted ventilation based on either the infant's characteristics or the duration of assisted ventilation required.

Among the 2462 audited infants, 1066 (43\%) experienced a specified transition during the 7 days prior to the audit. These transitions included admission, in-hospital transfers for surgery or diagnostic tests/procedures, or changes in status requiring a transition in the plan of care (i.e., initiation of antibiotics, extubation, initiation of pharmacologic treatment for NAS), as shown in Table 2. Of the 620 infants who were $\leq 7$ days at the time of the audit, the majority $(86 \%)$ were inborn and were admitted to the NICU directly from labor and delivery or from the local normal newborn unit 533 (86\%), and a minority (14\%) were admitted as transfers from an outside hospital.

The extent to which unit-level policies, guidelines, and procedures around transitions reflected the key drivers of communication, teamwork, standardization, and family integration is summarized by examining the hospital rate of the presence of policies and guidelines across the audit items aligned with these four key drivers. As shown in Fig. 2, hospitals had the greatest percentage of established policies and guidelines related to standardization (median:
Table 2 Frequency of infant transitions aside from provider handoffs.

Transition Infants with the transition
$n(\%)$

Admission from delivery room ${ }^{\mathrm{a}} \quad 533(86 \%)$

Admission via transport $^{\mathrm{a}} \quad 87(14 \%)$

Diagnostic test or procedure outside the $150(6 \%)$ NICU $^{\mathrm{b}}$

Surgery outside the NICU ${ }^{\mathrm{b}} \quad 94(4 \%)$

Started on antibiotics ${ }^{\mathrm{b}} \quad 567(23 \%)$

Extubated $^{\mathrm{b}} \quad 199(8 \%)$

Started pharmacologic therapy for $\quad 34(1 \%)$

$\mathrm{NAS}^{\mathrm{b}}$

${ }^{a}$ Denominator of $N=620$ infants $\leq 7$ days at time of audit.

${ }^{\mathrm{b}}$ Denominator of $N=2462$ infants.

$70 \%$ of audited policies in place) and teamwork (median: $67 \%$ of audited policies in place), while there were fewer established policies and guidelines for communication (median: $47 \%$ of audited policies in place) and family integration (median: $50 \%$ of audited policies in place). There was significant variation across hospitals, with some implementing $100 \%$ of the audited policies and guidelines and others implementing $<15 \%$ of the policies and guidelines. Detailed responses to the audit questions in each area are included in Supplementary Table 3.

\section{Communication}

The average hospital implemented $47 \%$ of the policies and guidelines reflective of good communication practices with the top quartile of hospitals implementing more than $65 \%$ and the bottom quartile implementing fewer than $35 \%$. Fifty-seven $(60 \%)$ of 95 centers used a structured communication tool for shift-to-shift handoffs. Eighty (92\%) of 87 centers that perform neonatal transports had systems to ensure immediate availability of medical advice for caregivers at referring hospitals with $23(26 \%)$ of these centers using telemedicine to communicate with referring providers. With respect to practices around the time of discharge, $88(93 \%)$ of 95 centers had a formal process to determine follow-up care needs and $83(87 \%)$ arranged home nursing visits for medically complex infants, but only $35(37 \%)$ centers created emergency intervention plans and notified emergency medical services providers at the time of discharge of a medically complex infant.

\section{Standardization}

The top quartile of hospitals in the area of standardization implemented at least $85 \%$ of the audited policies and guidelines supporting standardized processes around 
Fig. 2 Hospital variation in percent of policies, guidelines and procedures supporting communication, teamwork, standardization, and family integration. Hospital-level percent of audited unit-level policies, guidelines, and procedures that reflect key features of communication, teamwork, standardization, and family integration (minimum, 25th percentile, median, 75th percentile, maximum).

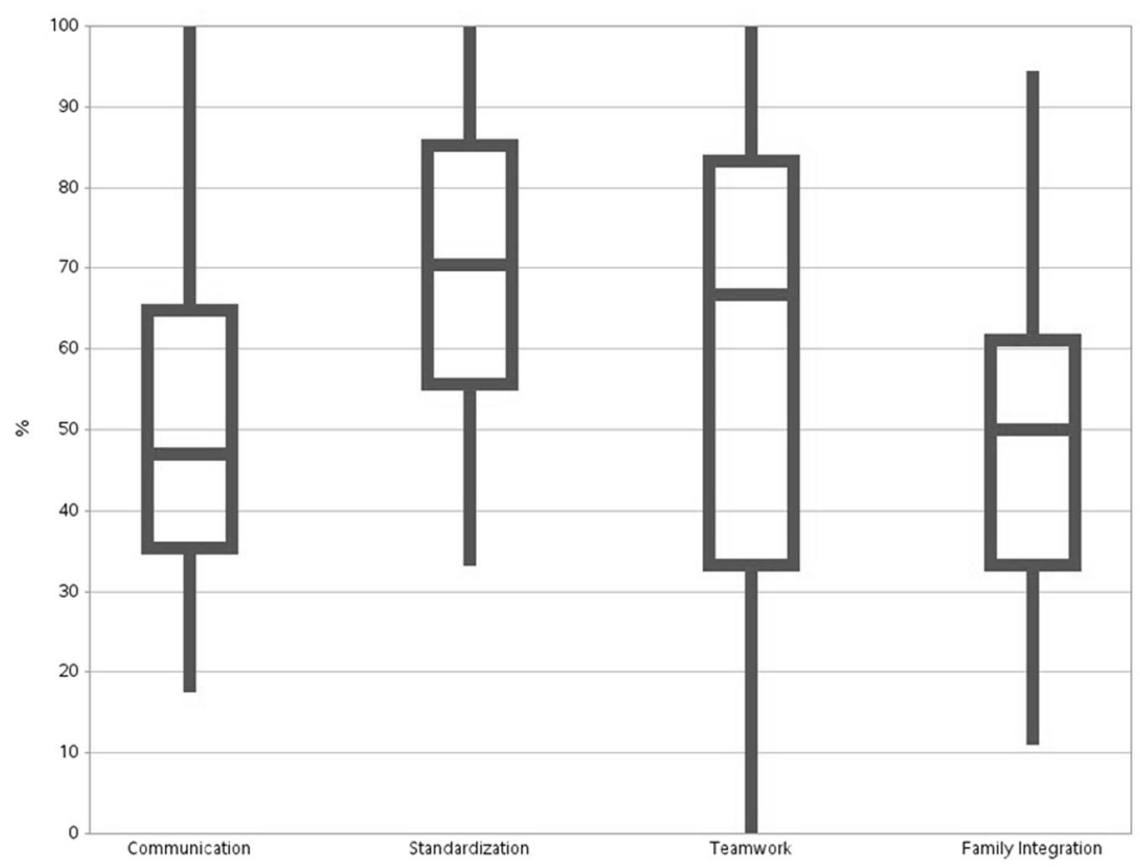

transitions, with a median hospital rate of $70 \%$. The bottom quartile of hospitals implemented fewer than $56 \%$ of the audited policies and guidelines related to standardization. Most of the 95 NICUs had formal guidelines regarding medical stability and clinical status for discharge including policies for adequacy of weight gain (59\%), thermal control (72\%), respiratory control (65\%), and feeding (64\%). Some centers used checklists to ensure necessary preparations for transporting infants to other areas of the hospital for diagnostic tests or procedures, with $50(57 \%)$ of 88 centers with MRI services using checklists for transfer for MRI and 39 $(66 \%)$ of the 59 centers with surgical services using checklists for transfer to the operating room.

\section{Teamwork}

Half of the participating centers implemented at least $67 \%$ of the practices and policies supporting effective teamwork. The top quartile of hospitals implemented more than $83 \%$ of the audited policies and guidelines and bottom quartile implemented fewer than $33 \%$. Only 44 $(46 \%)$ of 95 centers made teamwork training, such as TeamSTEPPS $^{\mathrm{TM}}$ or crew resource management training, available to all staff. Eighty-three (91\%) of the 91 centers with delivery services had programs for simulation-based neonatal resuscitation training and $60(66 \%)$ used delivery room equipment checklists. Fifty-three (60\%) of 88 centers with MRI services had written policies and guidelines regarding training requirements for personnel participating as part of the team transporting infants to diagnostic areas for magnetic resonance imaging and 33
(56\%) of 59 centers had similar guidelines for individuals participating in teams responsible for transport to the operating room.

\section{Family integration}

The median rate of hospital implementation of guidelines and policies supporting family integration in transitions was $50 \%$, with the top quartile implementing more than $61 \%$ of the policies and guidelines and the bottom quartile implementing fewer than $33 \%$ of the policies and guidelines. Of all 95 centers, 64 (67\%) had formal processes to engage families in planning transitions to home and 54 (57\%) assessed the adequacy and safety of the home environment. Among the 91 centers with delivery services, 27 (30\%) had policies and procedures regarding communication with families immediately after the delivery and $49(58 \%)$ of 87 centers with transport services had policies and procedures to assure communication with families on arrival at the receiving center.

\section{Discussion}

Among these 95 centers participating in the baseline audit of the iNICQ Improving Critical Transitions collaborative, sites documented changes in site of care, status, and plan of care during the NICU hospitalization among $43 \%$ of the 2462 infants audited. This is in addition to the frequent changes in the infant's team of providers that occur multiple times per day across several disciplines. While some 
policies and procedures within these units support good communication, teamwork, family integration, and standardization, practices that support these important drivers of optimal transitions are inconsistent and less than ideal. Hospitals had implemented the greatest percentage of policies and guidelines related to standardization and teamwork, while there was less implementation of the audited policies and guidelines for communication and family integration. There was significant variation across hospitals with some hospitals implementing $100 \%$ of the audited policies and guidelines and some hospitals implementing $<15 \%$ of the policies and guidelines.

The concept of "critical transitions" has been an invisible aspect of daily care in the NICU and has not been well studied, though some data suggest that poor transitions contribute to suboptimal outcomes. For example, studies have shown that poorly executed transitions of care at the time of discharge home can lead to increased length of stay, higher post-discharge healthcare utilization, and increased costs [28, 29]. Poor transitions likely also contribute to family dissatisfaction. A small qualitative study reported parents feeling distressed by the transfer of an infant to the NICU after birth and by the length of time they were separated from their infant and that even the transition from high-intensive to low-dependency care within the NICU is frightening for parents [30]. With respect to handoffs in the NICU, a recent study examining the impact of neonatologist continuity of care on short-term patient outcomes did not show any association between the number of neonatologist transitions and ventilator duration, oxygen use, blood stream infection, or urinary tract infections; however, similar to our results, they found that transitions were strikingly frequent [31]. We hypothesize that by focusing on implementation of evidence-based potentially better practices related to communication, teamwork, family integration, and standardization during transitions, we will be able to improve many of these important outcomes such as LOS, post-discharge healthcare utilization, family satisfaction, as well as key clinical outcomes.

This study is the first to describe and characterize NICU transitions using an action-oriented, improvement-focused logic model (key driver diagram). Historically the neonatal community has focused on important variations in mortality and the major morbidities of care. Much work has been done over the past 25 years to reduce the incidence of complications of prematurity and improve the quality of the processes and outcomes of care, yet gaps in quality remain [32, 33]. The current shift in focus, emphasizing smaller, but more frequent transitions of care represents an entirely new and fertile horizon for QI initiatives. The Joint Commission, Institute of Medicine and Agency for Healthcare Research and Quality have challenged hospitals to improve the effectiveness of transitions, with much of the current focus on the care of adults [15, 34, 35]. The opportunities for improvement uncovered in the baseline VON audit serve as a call to action to focus NICU QI efforts in this area. Furthermore, the iNICQ key driver diagram ties together important evidence from disparate literatures to identify a holistic set of key drivers and potential change ideas that may serve as a roadmap to improve critical transitions.

This study has a number of limitations. First, this was a cross-sectional structured assessment taking place in 95 units on 1 day in a 2 -week period. Therefore, assessments of patient-level care practices are limited by day-to-day variability in census, care providers, and patient acuity. In addition, the unit-level measures rely on self-report of the existence of policies, procedures, and guidelines, which may not reflect the actual existence of policies and guidelines, the quality of these policies, or compliance with these guidelines in practice. Sampling strategies for the patient-level audit varied among sites and may have affected our estimates of transition frequencies. The audit tool has not been validated and the assignment of questions to each of the four key drivers of the conceptual model was performed by the authors without formal psychometric testing. Therefore, it is possible that items we assigned to a construct (e.g., teamwork, communication, family integration), may in fact, be measuring something else or have overlap with other constructs. However, the authors feel that the assignment of items to these constructs (Supplementary Table 3) has face validity and is appropriate for such an exploratory analysis. The audit tool was designed with efficiency of large-scale implementation in mind, and was not intended to comprehensively measure all NICU transitions or to measure all types of transitions (changes in care team, site of care, or status/ plan of care) equally. In particular, policies and procedures related to changes in care team were addressed with the fewest items. However, the gaps between evidence and practice seen across audited transitions are likely to also be present in the other types of transitions. We believe that QI initiatives focused on processes surrounding transitions of care will result in better outcomes for NICU patients, but future analyses examining the links between transitionrelated processes and outcomes will be required to test this hypothesis.

This study adds to what little is currently known about NICU transitions. The results from the VON iNICQ baseline audit and the upcoming work that will be conducted as part of a multi-year QI collaborative will greatly add to our improved understanding of how to optimize the transitions of care frequently experienced by newborns and their families. 
Funding RFS, JDH, and KAM are employees of the Vermont Oxford Network (VON). MEB-F was employed by VON during the conduct of this work. EME is funded by a grant from VON to the University of Vermont. HCK, JM, WT, HC, and MF have served as faculty for VON.

Author contributions HCK substantially contributed to the conception and design of the work, analyzed and interpreted the data, drafted the initial manuscript, created the tables and figures, and critically revised the manuscript for important intellectual content. EME and KAM substantially contributed to the analysis and interpretation of the data, assisted in creation of tables and figures, and critically revised the manuscript for important intellectual content. JM, WT, HC, ES, RFS, MEB-F, JDH, and MF substantially contributed to the conception and design of the work and critically revised the manuscript for important intellectual content.

\section{Compliance with ethical standards}

Conflict of interest The authors declare that they have no conflict of interest.

Publisher's note Springer Nature remains neutral with regard to jurisdictional claims in published maps and institutional affiliations.

\section{References}

1. Gray JE, Davis DA, Pursley DM, Smallcomb JE, Geva A, Chawla NV. Network analysis of team structure in the neonatal intensive care unit. Pediatrics. 2010;125:e1460-7.

2. Edwards EM, Ehret DEY, Soll RF, Horbar JD. Vermont Oxford Network: a worldwide learning community. Transl Pediatr. 2019;8:182-92.

3. Beck AF, Edwards EM, Horbar JD, Howell EA, McCormick MC, Pursley DM. The color of health: how racism, segregation, and inequality affect the health and well-being of preterm infants and their families. Pediatr Res. 2020;87:227-34.

4. Stevenson DK, Wong RJ, Profit J, Shaw GM, Jason Wang C, Lee HC. "Following through": addressing the racial inequality for preterm infants and their families. Pediatr Res. 2020;87:192-3.

5. Horbar JD, Soll RF, Edwards WH. The Vermont Oxford Network: a community of practice. Clin Perinatol. 2010;37:29-47.

6. Institute of Medicine. Crossing the quality chasm: a new health system for the 21st century. 1st ed. Washington, DC: National Academies Press; 2001.

7. Little GA, Horbar JD, Wachtel JS, Gluck PA, Muri JH. Evolution of quality improvement in perinatal care in toward improving the outcome of pregnancy III. 2010. https://www.marchofdimes.org/ toward-improving-the-outcome-of-pregnancy-iii.pdf.

8. Riley W, Davis S, Miller K, Hansen H, Sainfort F, Sweet R. Didactic and simulation nontechnical skills team training to improve perinatal patient outcomes in a community hospital. Jt Comm J Qual Patient Saf. 2011;37:357-64.

9. Salas E, Cooke NJ, Rosen MA. On teams, teamwork, and team performance: discoveries and developments. Hum Factors. 2008;50:540-7.

10. Salas E, Gregory ME, King HB. Team training can enhance patient safety-the data, the challenge ahead. Jt Comm J Qual Patient Saf. 2011;37:339-40.

11. Shuffler ML, DiazGranados D, Salas E. There's a science for that: team development interventions in organizations. Curr Directions Psychological Sci. 2011;20:365-72.
12. TeamSTEPPS. TeamSTEPPS 2.0 Pocket guide: team strategies \& tools to enhance performance and patient safety. AHRQ Pub No 14-0001-2. Rockville, MD: AHRQ; 2013.

13. DeMauro SB, Douglas E, Karp K, Schmidt B, Patel J, Kronberger A, et al. Improving delivery room management for very preterm infants. Pediatrics. 2013;132:e1018-25.

14. Katheria A, Rich W, Finer N. Development of a strategic process using checklists to facilitate team preparation and improve communication during neonatal resuscitation. Resuscitation. 2013;84:1552-7.

15. The Joint Commission. Inadequate hand-off communication. Sentinel Event Alert, Issue 58, September 12, 2017. https://www. jointcommission.org/assets/1/18/SEA_58_Hand_off_Comms_9_ 6_17_FINAL_(1).pdf. Accessed 6 Nov 2019.

16. Albritton J, Maddox L, Dalto J, Ridout E, Minton S. The effect of a newborn telehealth program on transfers avoided: a multiplebaseline study. Health Aff (Millwood). 2018;37:1990-6.

17. Balakrishnan M, Raghavan A, Suresh GK. Eliminating undesirable variation in neonatal practice: balancing standardization and customization. Clin Perinatol. 2017;44:529-40.

18. Luria JW, Muething SE, Schoettker PJ, Kotagal UR. Reliability science and patient safety. Pediatr Clin North Am. 2006;53:1121-33.

19. Pronovost PJ, Berenholtz SM, Goeschel CA, Needham DM, Sexton JB, Thompson DA, et al. Creating high reliability in health care organizations. Health Serv Res. 2006;41:1599-617.

20. Rozich JD, Howard RJ, Justeson JM, Macken PD, Lindsay ME, Resar RK. Standardization as a mechanism to improve safety in health care. Jt Comm J Qual Saf. 2004;30:5-14.

21. Starmer AJ, Spector ND, Srivastava R, West DC, Rosenbluth G, Allen $\mathrm{AD}$, et al. Changes in medical errors after implementation of a handoff program. N Engl J Med. 2014;371:1803-12.

22. Petersen LA, Orav EJ, Teich JM, O’Neil AC, Brennan TA. Using a computerized sign-out program to improve continuity of inpatient care and prevent adverse events. Jt Comm J Qual Improv. 1998;24:77-87.

23. Bate P, Robert G. Experience-based design: from redesigning the system around the patient to co-designing services with the patient. Qual Saf Health Care. 2006;15:307-10.

24. The Point of Care Foundation. Carrying out observations. https://www.pointofcarefoundation.org.uk/resource/experience-ba sed-co-design-ebcd-toolkit/step-by-step-guide/5-carrying-observa tions/. Accessed 29 Nov 2018.

25. Patrick SW, Schumacher RE, Horbar JD, Buus-Frank ME, Edwards EM, Morrow KA, et al. Improving care for neonatal abstinence syndrome. Pediatrics. 2016;137:e20153835.

26. Hagadorn JI, Sink DW, Buus-Frank ME, Edwards EM, Morrow KA, Horbar JD, et al. Alarm safety and oxygen saturation targets in the Vermont Oxford Network iNICQ 2015 collaborative. J Perinatol. 2017:37:270-6.

27. Ho T, Buus-Frank ME, Edwards EM, Morrow KA, Ferrelli K, Srinivasan A, et al. Adherence of newborn-specific antibiotic stewardship programs to $\mathrm{CDC}$ recommendations. Pediatrics. 2018;142:e20174322.

28. Kuzniewicz MW, Parker SJ, Schnake-Mahl A, Escobar GJ. Hospital readmissions and emergency department visits in moderate preterm, late preterm, and early term infants. Clin Perinatol. 2013;40:753-75.

29. Underwood MA, Danielsen B, Gilbert WM. Cost, causes and rates of rehospitalization of preterm infants. J Perinatol. 2007;27:614-9.

30. Gavey J. Parental perceptions of neonatal care. J Neonatal Nurs. 2007;13:199-206.

31. Machut KZ, Datta A, Stoller JZ, Rao R, Mathur A, Grover TR, et al. Association of neonatologist continuity of care and shortterm patient outcomes. J Pediatr. 2019. 
32. Horbar JD, Edwards EM, Greenberg LT, Morrow KA, Soll RF, Buus-Frank ME, et al. Variation in performance of neonatal intensive care units in the United States. JAMA Pediatr. 2017;171: e164396.

33. Spitzer AR. Has quality improvement really improved outcomes for babies in the neonatal intensive care unit? Clin Perinatol. 2017;44:469-83.
34. The Joint Commission. The Joint Commission transitions of care (ToC) portal. https://www.jointcommission.org/toc.aspx. Accessed 12 Aug 2019.

35. The Joint Commission. Hot topics in health care: transitions of care: the need for a more effective approach to continuing patient care. https://www.jointcommission.org/assets/1/18/Hot_Topics_ Transitions_of_Care.pdf. Accessed 11 Sept 2019. 